\title{
A PARTICIPAÇÃO DE JOVENS COM DEFICIÊNCIA VISUAL EM AULAS DE EDUCAÇÃO FÍSICA: EXPERIÊNCIAS NA REDE REGULAR E EM INSTITUIÇÕES ESPECIALIZADAS ${ }^{1}$
}

\author{
Otávio Luis Piva da Cunha Furtado \\ Universidade de São Paulo, Ribeirão Preto, São Paulo, Brasil. \\ Márcio Pereira Morato \\ Universidade de São Paulo, Ribeirão Preto, São Paulo, Brasil. \\ Gustavo Luis Gutierrez \\ Universidade Estadual de Campinas, Campinas, São Paulo, Brasil. \\ Maria Luíza Tanure Alves \\ Universidade Estadual de Campinas, Campinas, São Paulo, Brasil.
}

\begin{abstract}
Resumo
Este estudo buscou analisar a participação de alunos cegos e com baixa visão em aulas de Educação Física. A coleta de dados contou com aplicação de questionário a 50 jovens praticantes de goalball (27 meninas), com idade entre 13 e 19 anos. Os achados apontaram acentuada redução da participação nas aulas de Educação Física na rede regular na medida em que aumentava a limitação visual. As principais barreiras destacadas foram: a falta de colaboração dos colegas, o medo de se machucar, a falta de capacitação dos professores. A prática de modalidades paralímpicas foi predominante nas instituições de ensino especializado, enquanto na rede regular somente modalidades coletivas convencionais eram praticadas. Tal cenário, portanto, não corresponde ao ideal de inclusão educacional.
\end{abstract}

Palavras-chave: Transtornos da visão. Exercício. Educação Física e treinamento.

\section{THE ENGAGEMENT OF YOUTH WITH VISUAL IMPAIRMENTS IN PHYSICAL EDUCATION CLASSES: EXPERIENCES IN REGULAR SCHOOLS AND IN SPECIALIZED INSTITUTIONS}

\begin{abstract}
This study analyzed the engagement of youth with visual impairments in physical education classes. Data collection included the application of a questionnaire to 50 young goalball players (27 girls), aged 13 to 19 years. Our findings showed a marked reduction of participation in the regular Physical Education classes as visual deficits increased. The main barriers mentioned were the lack peers collaboration, the fear of being hurt, and the lack of teachers' training. The practice of Paralympic sports was predominant in specialized schools, whereas in the regular schools only conventional team sports were practiced. Such a scenario, therefore, does not correspond to the inclusion ideal.
\end{abstract}

Keywords: Vision Disorders. Exercise. Physical Education and Training.

1 Apoio financeiro: Coordenação de Aperfeiçoamento de Pessoal de Nível Superior (CAPES). 


\title{
LA PARTICIPACIÓN DE JÓVENES CON DISCAPACIDAD VISUAL EN CLASES DE EDUCACIÓN FÍSICA: EXPERIENCIAS EN EL SISTEMA REGULAR Y EN INSTITUCIONES ESPECIALIZADAS
}

\begin{abstract}
Resumen
Este estudio analizó la participación de alumnos ciegos y de baja visión en las clases de Educación Física. La recolección de datos incluyó la aplicación de un cuestionario a 50 jóvenes jugadores de goalball (27 niñas), de 13 a 19 años. Identificamos una marcada reducción de la participación en las clases regulares de educación física a medida que los déficits visuales progresaron a la limitación visual. Las principales barreras mencionadas fueron la falta de colaboración de outros alumnos, el miedo de herirse y la falta de formación del profesorado. La práctica de deportes paraolímpicos predominó en las Instituciones de Enseñanza Especializada, mientras que en las escuelas regulares sólo practicaban deportes convencionales. Este escenario, por lo tanto, no corresponde al ideal de inclusión educativa.
\end{abstract}

Palabras-clave: Trastornos de la Visión. Ejercicio. Educación y Entrenamiento Físico.

\section{Introdução}

O movimento inclusivo defende que crianças com deficiência devem ser incluídas no ensino regular, para que possam se desenvolver e participar ativamente da sociedade (ODOM; DIAMOND, 1998; STAINBACK; STAINBACK, 1999; MARCHESI, 2001; RODRIGUES, 2001; PIVIK; MCCOMAS; LAFLAME, 2002). Seu processo educacional deve respeitar suas diferenças e atender às suas necessidades educacionais como um todo, permitindo que essas crianças sejam um membro ativo dentro de sua escola (PLACE; HODGE, 2001; KODISK et al., 2006).

De acordo com a legislação educacional brasileira, o aluno com deficiência tem garantido o seu direito a ter educação de qualidade no sistema regular de ensino. Esse direito está assegurado, entre outros documentos, pela Lei de Diretrizes e Bases, Plano Nacional de Educação, Decreto-lei 3298/1999, Decreto 186, de 2008, que aprova o texto da Convenção sobre os Direitos das Pessoas com Deficiência, e também pela Política Nacional de Educação Especial na Perspectiva da Educação Inclusiva (BRASIL, 1996; 1999; 2001b; 2008a; 2008b). Tais documentos descrevem que o aluno com deficiência tem direito à educação de qualidade no sistema regular de ensino, com a reestruturação das escolas e a capacitação profissional para o atendimento das suas necessidades educacionais.

De acordo com o Censo Escolar do Instituto Nacional de Estudos e Pesquisas Educacionais (INEP) de 2011, 80\% das matrículas de alunos com deficiência se encontravam nas escolas públicas, enquanto os $20 \%$ restantes estão nas escolas privadas. O censo também reafirmou a tendência de crescimento do número de matrículas de alunos com deficiência no ensino regular, com incremento de 15,3\%, de 2010 para 2011. Nesse âmbito, 95,2\% das crianças de 6 a 14 anos com deficiência frequentam a escola. Os adolescentes com 15 anos ou mais e com alguma deficiência apresentam uma taxa de alfabetização de 81,7\% (INEP, 2012). No entanto, frequentar o ensino regular ainda não é garantia de inclusão.

Atualmente, o conceito de inclusão educacional ultrapassa o atendimento das necessidades de alunos com deficiência, compreendendo o oferecimento de um ensino de qualidade para todos os alunos (BOOTH; AINSCOWN, 2002). A inclusão na escola perpassa pelo reconhecimento do conceito de desvantagem, relacionado ao prejuízo do indivíduo para exercer seus papéis sociais em decorrência de restrições no ambiente e de exigências da tarefa diante de suas necessidades individuais (AMIRALIAN et al., 2000). O reconhecimento da 
desvantagem como fator principal no processo inclusivo permite que o professor reconheça as necessidades do grupo, promovendo adaptações na tarefa e no ambiente para a melhor participação de todos (MITTLER, 2003).

Contudo, apesar de resultados positivos já indicados a favor da inclusão de alunos com deficiência (BLOCK; OBRUSNIKOVA, 2007; GRENIER; COLLINS; WRIGHT, 2014), estes ainda enfrentam obstáculos importantes para a sua inclusão nas aulas de Educação Física (EF) (COATES; VICKERMAN, 2008; QI; HA, 2012; HAEGELE; SUTHERLAND, 2015). As dificuldades estão relacionadas principalmente à falta de adaptações adequadas nas atividades de acordo com as necessidades do aluno, de acessibilidade ao local da aula e de material adaptado, à participação segregada durante as aulas, bem como a não aceitação do aluno com deficiência pela turma (BLINDE; MCCALLISTER, 1998; GOODWIN; WATKINSON, 2000; SPENCER-CAVALIERE; WATKINSON, 2010; ALVES; DUARTE, 2013; BREDAHL, 2013; ALVES; DUARTE, 2014; HAEGELE; SUTHERLAND, 2015).

Nesse contexto, é importante reconhecer que a EF escolar tem como ponto fundamental o conceito de habilidade e performance, construído socialmente sobre um padrão de normalidade e pela valorização do corpo hábil, forte e capaz (BARTON, 1993; EVANS, 2004; FITZGERALD, 2005). O conceito de habilidade, associado ao conceito social de ser capaz, é o cerne do esporte e de atividades físicas (DEPAUW, 1997). Dessa forma, alunos com deficiência não conseguem responder às demandas das aulas de EF e são julgados através do olhar da habilidade e da performance, contribuindo para a falta de participação e o isolamento do grupo (ALVES; DUARTE, 2013; HAEGELE; SUTHERLAND, 2015).

As aulas de EF são estruturadas sobre práticas corporais e esportes construídos para pessoas sem deficiência. Essas práticas são afirmadas nos conteúdos curriculares propostos a cada nível de ensino, nos quais estão presentes os esportes, a ginástica, as lutas, a dança e os jogos. Apesar do direito à inclusão, os conteúdos curriculares da EF escolar ainda não trazem práticas e esportes relacionados especificamente aos alunos com deficiência. Por tais motivos, autores da área da Educação Física Adaptada questionam os conhecimentos escolhidos e os valores presentes na aula de EF em face do aluno com deficiência (BARTON, 1993; BRITTAIN, 2004a; 2004b; EVANS, 2004; FITZGERALD, 2005; COSTA; MUNSTER, 2017).

Nesse âmbito, é importante reconhecer que as relações instituídas nas aulas de EF entre o aluno com deficiência, seus colegas e os conteúdos curriculares devem ser analisados dentro de um contexto social amplo, reconhecendo valores, comportamentos e crenças construídas culturalmente. Dessa forma, o presente estudo busca analisar a participação de adolescentes cegos e com baixa visão nas aulas de EF no ensino regular e descrever as práticas esportivas realizadas nesse ambiente e em instituições especializadas para atendimento a pessoas com deficiência visual.

\section{Método}

\section{Participantes}

Participaram deste estudo 50 jovens praticantes de goalball $(27$ meninas e 22 meninos), com idade entre 13 e 19 anos, abrangendo $78 \%$ (34 meninas e 30 meninos) do total de inscritos nessa modalidade na edição de 2012 das Paralimpíadas Escolares (Tabela 1). Tal evento tem sido realizado anualmente pelo Comitê Paralímpico Brasileiro e tem como objetivo aumentar a prática de esportes paralímpicos por estudantes com deficiência física, visual e intelectual.

Os critérios de inclusão dos participantes foram: 1) fazer parte de uma das equipes de goalball inscritas; 2) estar regularmente matriculado no ensino fundamental ou médio; 3) ter 
idade entre 13 e 19 anos, e 4) ter sido considerado elegível de acordo com a classificação oftalmológica reconhecida pelo Comitê Paralímpico Brasileiro (CPB). A classificação visual esportiva foi determinada por um classificador visual internacional (médico oftalmologista), seguindo as diretrizes da United States Association of Blind Athletes. O sistema é dividido em três classes: B1-sem percepção visual em ambos os olhos até percepção luminosa, contanto que não consigam reconhecer a forma de uma mão a qualquer distância ou em qualquer direção; B2 - capacidade de reconhecer a forma de uma mão até a acuidade visual de 2/60 e ou o campo visual de menos de 5 graus no melhor olho após correção; B3- acuidade visual acima de 2/60 até 6/60 e ou um campo visual de menos de 20 graus no melhor olho após correção (FURTADO et al., 2015).

Tabela 1. Caracterização da amostra

\begin{tabular}{|c|c|c|c|}
\hline & \multicolumn{3}{|c|}{ Classificação Visual } \\
\hline & $\begin{array}{c}\mathrm{B} 1 \\
(N=15)\end{array}$ & $\begin{array}{c}\mathrm{B} 2 \\
(N=20)\end{array}$ & $\begin{array}{c}\mathrm{B} 3 \\
(N=15)\end{array}$ \\
\hline \multicolumn{4}{|l|}{ Gênero } \\
\hline Feminino $(N=27)$ & 9 & 11 & 7 \\
\hline Masculino $(N=23)$ & 6 & 9 & 8 \\
\hline Idade (anos) & $17,1( \pm 1,7)$ & $17,4( \pm 1,2)$ & $17,0( \pm 1,4)$ \\
\hline Escolaridade (anos) & $9,7( \pm 2,1)$ & $9,5( \pm 2,3)$ & $9,3( \pm 2,2)$ \\
\hline
\end{tabular}

Fonte: Elaborado pelos autores, 2018.

Do total amostral, 15 atletas estudavam no ensino fundamental II e 35 no ensino médio quando a pesquisa foi realizada. Tendo em vista as diferentes propostas curriculares e metodológicas possíveis entre o ensino fundamental e o médio, os participantes foram questionados sobre suas experiências vividas durante o ensino fundamental II. Durante esse nível de ensino, dez alunos estudavam exclusivamente em Instituições Especializadas (IE) de atendimento a alunos com deficiência visual, onde tinham aulas de EF com professores especializados; quatro estudavam concomitantemente na Rede Regular (RR) e em IE e não participavam das aulas de EF na RR devido à apresentação de atestado de frequência nas aulas de EF na IE; 36 estudavam na RR, sendo que 24 participavam de, pelo menos, alguma atividade nas aulas de EF.

O estudo foi aprovado por um Comitê de Ética em Pesquisa (Faculdade de Ciências Médicas - Universidade Estadual de Campinas). Antes da competição, uma carta convite eletrônica foi enviada para os chefes de delegação de cada estado, explicando os objetivos e os métodos de pesquisa. Os participantes maiores de 18 anos assinaram um termo de consentimento livre e esclarecido. Àqueles com idade inferior a 18 anos foram solicitados seu assentimento e a autorização do respectivo responsável legal.

\section{Procedimentos}

O roteiro de entrevista teve sua validade de conteúdo confirmada por dois especialistas em Educação Física Adaptada, cada um com mais de dez anos de experiência em pesquisa e docência em ensino superior. Os especialistas revisaram e proporcionaram feedback sobre os elementos do questionário (HAYNES; RICHARD; KUBANY, 1995). O instrumento foi 
aplicado em teste piloto com 11 atletas de goalball durante edição anterior do evento, permitindo seu refinamento quanto à clareza das questões e a inclusão de itens de resposta para maior agilidade na sua aplicação.

O instrumento abordou a caracterização dos participantes quanto ao contexto das aulas de EF, com questões a respeito da sua participação na aula de EF; de obstáculos presentes nas aulas e que dificultavam sua participação; estratégias de ensino utilizadas pelo professor de EF; práticas propostas na RR e na IE, e adaptações promovidas pelos professores de EF.

Três pesquisadores com formação em EF e experiência prática em Educação Física Adaptada aplicaram os questionários ao longo de três dias. Após autorização de seu respectivo(a) técnico(a), os participantes eram convidados a participar do estudo, com duração entre 15 e 30 minutos. As entrevistas ocorreram em momentos de ócio dos participantes, no hotel onde estavam hospedados ou quando estavam nas arquibancadas do local de competição. Optamos por não realizar a pesquisa em períodos do dia em que a equipe tivesse atividade em quadra, durante jogos da equipe do mesmo estado (do outro gênero) ou em momentos decisivos do campeonato, como em disputas de jogos eliminatórios e finais. Acreditamos que a atenção dos atletas poderia estar reduzida e voltada para outra situação, interferindo na resposta aos questionamentos.

\section{Análise dos dados}

Neste estudo, empregamos uma abordagem mista (quantitativa e qualitativa) para a análise e a apresentação dos dados. A estatística descritiva foi utilizada para a apresentação dos dados relativos ao gênero, à idade e à escolaridade da amostra, bem como para a caracterização do local de ensino dos participantes do estudo e das atividades por eles praticadas nas aulas de EF. O teste Qui-Quadrado $(p<.05)$ foi usado para a análise do grau de participação nas aulas práticas de EF no Ensino Fundamental II na RR. As perguntas discursivas foram analisadas em duas fases: o agrupamento e a apresentação das respostas, e a escrita e a verificação de conclusões. A apresentação das respostas em formato visual (planilha de dados) permitiu categorizar os dados, auxiliando na detecção de diferenças e similaridades entre temas e subtemas, com o consequente desenvolvimento de asserções (MILES; HUBERMAN; SALDAÑA, 2014).

\section{Resultados}

Quando desconsideradas as classes esportivas, não encontramos diferença quanto ao grau de participação de alunos com deficiência visual nas aulas de EF no ensino fundamental II da RR, $X^{2}(3), 2,22, p=0,528$, com participação distribuída entre: todas atividades (28\%); maioria das atividades (17\%); algumas atividades (22\%); não participava (33\%). Por outro lado, a comparação entre grupos indicou que alunos com classificação esportiva B3 participavam significantemente mais de "todas as atividades" durante as aulas de EF (Tabela 2).

Tabela 2. Participação nas aulas práticas de Educação Física na Rede Regular

\begin{tabular}{lccccc}
\hline & $\begin{array}{c}\text { B1 } \\
(\mathbf{n = 9 )}\end{array}$ & $\begin{array}{c}\mathbf{B 2} \\
(\mathbf{n = 1 6})\end{array}$ & $\begin{array}{c}\text { B3 } \\
(\mathbf{n = 1 1})\end{array}$ & $\begin{array}{c}\text { Fisher' } \\
\mathbf{s} \\
\text { test }\end{array}$ & $\begin{array}{c}\text { Valor } \\
\mathbf{d e} \boldsymbol{p}\end{array}$ \\
\hline Todas as atividades & $11 \%$ & $13 \%$ & $64 \%$ & 9,78 & 0,008 \\
\hline Maioria das atividades & $11 \%$ & $25 \%$ & $9 \%$ & 1,47 & 0,481 \\
\hline Algumas atividades & $44 \%$ & $19 \%$ & $9 \%$ & 3,63 & 0,163 \\
\hline Não participava & $33 \%$ & $44 \%$ & $18 \%$ & 2,01 & 0,366
\end{tabular}

Fonte: Elaborado pelos autores, 2018. 
Os motivos relatados para não participarem das aulas de EF referem-se principalmente à falta de colaboração dos colegas, ao medo de se machucar em atividades com bola, à falta de capacitação dos professores e às necessidades de adaptação do espaço. Como alternativa para compensar sua não participação nas aulas, alguns alunos entregavam trabalhos escritos. Cabe destacar que, em quatro casos, os participantes não souberam ou preferiram não elencar motivos para a sua exclusão das aulas de EF na RR.

Dentre os 24 alunos que participaram de ao menos alguma atividade nas aulas de EF na RR, constatamos uma tendência crescente de relato de ausência de dificuldade em participar das aulas, na medida em que a capacidade visual dos participantes aumentava (B1: 17\%; B2: 25\%; B3: 33\%). Em 50\% dos casos de alunos com classificação B2 e em 89\% dos casos de alunos com classificação B3, não havia emprego de qualquer estratégia adicional de ensino por parte dos professores. Por vezes, para explicar uma atividade, o professor se aproximava dos alunos com baixa visão ou mesmo demonstrava movimentos por meio de manipulação. Com maior frequência, o professor adaptava as atividades para alunos com cegueira ou utilizava um colega tutor durante as aulas.

Com relação às atividades praticadas pelos participantes, além da frequente inclusão nas sessões de alongamento e, eventualmente, em jogos e brincadeiras, observamos um contraste entre as atividades realizadas no ensino na RR em comparação àquelas praticadas em IE. Enquanto no instituto a prática de modalidades paralímpicas era predominante, no ensino regular as modalidades coletivas convencionais eram mais frequentes (Tabela 3 ).

Tabela 3. Modalidades esportivas praticadas nas aulas de Educação Física na Rede Regular e no Instituto Especializado

\begin{tabular}{llll}
\hline \multicolumn{1}{c}{ Rede Regular $(\mathbf{N}=\mathbf{2 4})$} & \multicolumn{2}{c}{ Instituto $(\mathbf{N = 1 4 )}$} \\
\hline Futsal & $83 \%$ & Goalball & $100 \%$ \\
\hline Vôlei & $46 \%$ & Natação & $50 \%$ \\
\hline Basquete & $33 \%$ & Ginástica & $29 \%$ \\
\hline Atletismo & $33 \%$ & Atletismo & $29 \%$ \\
\hline Handball & $21 \%$ & Futsal & $29 \%$ \\
\hline Ginástica & $13 \%$ & Futebol de 5 & $21 \%$ \\
\hline Karatê & $4 \%$ & Judô & $14 \%$ \\
\hline Natação & $4 \%$ & & \\
\hline
\end{tabular}

Nota: cada participante citou mais de uma atividade durante a entrevista.

Fonte: Elaborado pelos autores, 2018.

\section{Discussão}

Neste estudo, analisamos a participação de adolescentes cegos e com baixa visão nas aulas de EF no ensino fundamental II da RR e descrevemos as práticas esportivas oferecidas a eles nesse ambiente e em IE para pessoas com deficiência visual. A falta de colaboração dos colegas, o medo de se machucar em atividades com bola, a falta de capacitação dos professores e as necessidades de adaptação do espaço foram os motivos destacados como barreiras à participação nas aulas de EF. Os participantes com maior capacidade visual relataram maior participação nas aulas, apresentando menores dificuldades na prática e na adaptação de estratégias de ensino pelos professores. A prática de modalidades paralímpicas foi predominante nas IE, enquanto na $R R$ as modalidades coletivas convencionais se mostraram mais frequentes.

Dentre as funções sensoriais humanas, a visão é notoriamente a mais empregada para nos relacionarmos com o mundo externo. No âmbito das aulas de EF escolar, embora o tato e 
a audição tenham função importante para facilitar a participação de alunos nas atividades propostas, é a informação visual que, via de regra, permite ao aluno perceber o ambiente da prática, os materiais pedagógicos e os modelos de aprendizagem proporcionados pelos pares ou por professores (STEWART et al., 2015). Talvez por esse fato, os participantes com maior função visual tenham participado consideravelmente mais de atividades nas aulas de EF em relação àqueles com menor capacidade visual. Quase dois terços dos participantes com classificação visual B3 (menor grau de deficiência visual) relataram participar de todas as atividades na EF na RR, enquanto seus pares com classificação B2 e B1 participavam proporcionalmente cinco a seis vezes menos, respectivamente. Tanto a limitação de aprendizagem incidental (observando o ambiente e repetindo) quanto a dificuldade de adoção de estratégias não visuais pelos professores parecem ter importante papel nessa questão (MUNSTER; ALMEIDA, 2005) e merecem ser melhor esclarecidas em estudos futuros.

A exclusão ou a participação limitada de alunos com deficiência visual das aulas de EF também são encontradas em estudos anteriores (ALVES; DUARTE, 2013; 2014). De forma geral, os motivos elencados para a não participação relacionam-se ao não reconhecimento das necessidades educacionais desse aluno diante das exigências da aula de EF. Nesse sentido, a compreensão do processo inclusivo de alunos com deficiência visual nas aulas de EF inclui não só a questão do déficit visual, mas, sobretudo, a cultura e as demandas características do contexto das aulas de EF (FIORINI; NABEIRO, 2013; FITZGERALD, 2005).

Os conteúdos da EF na RR são normalmente construídos socialmente e refletem a cultura na qual estão inseridos. Por isso, precisam ser entendidos em termos de relações de poder, privilégio e dominação (SAGE, 1993). De modo geral, os participantes deste estudo relataram dificuldade de engajamento em atividades sem adaptações de acordo com as suas necessidades. Representado pelo currículo proposto nas aulas, pode-se observar a dificuldade de grande parte dos participantes deste estudo em responder às exigências das aulas de EF.

Durante as aulas de EF, os alunos com deficiência se deparam com um currículo estruturado para o aluno sem deficiência. $\mathrm{O}$ que pode ser confirmado pelos dados deste estudo, que indicam a predominância das modalidades futsal (83\%) e vôlei (46\%). Dessa forma, sua participação acaba sendo balizada pela capacidade de responder às exigências das atividades (BARTON, 1993; BRITTAIN, 2004a; 2004b; EVANS, 2004; FITZGERALD, 2005), o que compromete seu envolvimento ativo, principalmente na medida em que sua limitação visual aumenta.

No caso da participação em aulas de EF na IE, jovens alunos com deficiência visual têm atendimento de profissional especialista. Para atender às características desse alunado, as atividades são coerentes com suas capacidades e limitações (Tabela 3). Neste estudo, entrevistamos praticantes de goalball que, matriculados em uma IE, relataram praticar a modalidade ao longo dos semestres letivos, com frequência de duas a três vezes por semana. Em outros estudos, a prática regular de goalball mostrou-se positiva para que jovens com deficiência visual atingissem níveis satisfatórios de aptidão física (KARAKAYA; AKI; ERGUN, 2009; FURTADO et al., 2016). Níveis elevados de aptidão física estão associados à prática regular de atividades físicas, que, por sua vez, proporcionam um perfil positivo de saúde. Embora a Organização Mundial da Saúde (World Health Organization - WHO) recomende para crianças e adolescentes o acúmulo de 60 minutos diários de atividade física de moderada e alta intensidade, é recomendado o acúmulo proveniente de múltiplas atividades e em diferentes ambientes, como o escolar, o comunitário e o familiar (WHO, 2010).

Nossos dados indicam que, enquanto os participantes matriculados na IE praticavam, além do goalball, outras modalidades que complementavam sua prática corporal, na EF na RR os alunos com classificação B3 parecem ser os mais beneficiados, pois participaram em sua maioria de "Todas as atividades". Cabe, contudo, apontar que a melhora de aspectos da 
saúde deve ser apenas um dos motivos para jovens praticarem atividades físicas regularmente. As aulas de EF têm potencial de fomentar o desenvolvimento integral dos estudantes, contribuindo para a apropriação crítica da cultura corporal de movimento (BETTI; ZULIANI, 2002), justificando, assim, a importância de uma EF inclusiva.

Atualmente, a aula de EF, como está estruturada culturalmente, não assume a diversidade entre os alunos e a desvantagem existente diante de práticas corporais homogêneas. Estruturados sobre o conceito de cultura corporal de movimento, os conteúdos e as práticas estabelecidas nas aulas de $\mathrm{EF}$ desconsideram que a cultura corporal de crianças e jovens com deficiência é diferente. A escolha de determinados conteúdos curriculares em detrimento de outros revela valores presentes no contexto educacional. A escola, como espaço de legitimação da cultura da classe dominante, reconhece os esportes convencionais como os conteúdos curriculares únicos e legítimos a serem transmitidos aos alunos (BARTON, 1993). O esporte paralímpico não é reconhecido como parte desse capital cultural legítimo (BRITTAIN, 2004a; 2004b), sendo restrito a ambientes exclusivos de pessoas com deficiência, como associações e institutos que atendem pessoas com determinada condição, tal qual demonstrado em nossos achados. Embora iniciativas que promovam a vivência dos esportes paralímpicos para todos na escola venham sendo exploradas, como é o caso do Dia Paralímpico (BORGMANN; ALMEIDA, 2015), estas ainda são incipientes.

A aula de EF especificamente centrada na prática esportiva de modalidades coletivas convencionais nega a cultura corporal e as habilidades de alunos com deficiência. Mais do que adaptações em atividades e materiais, o conceito de inclusão exige compreender que a cultura escolar não pertence a um grupo específico, mas sim a todos os alunos. Essas mudanças exigem alterações curriculares importantes, especificamente no caso das aulas de EF na RR (COSTA; MUNSTER, 2017). Alunos com e sem deficiência devem ter contato com a cultura corporal como um todo e não somente àquela restrita aos critérios de habilidade e performance voltados à pessoa sem deficiência. Nesse contexto, os esportes adaptados e/ou paralímpicos devem fazer parte da cultura presente nas aulas de EF, eminentemente naquelas ofertadas na RR. Assim como alunos sem deficiência têm na escola o local de direito para conhecer e vivenciar as modalidades esportivas, alunos com deficiência também devem ter esse direito garantido.

É importante destacar algumas limitações deste estudo. Em primeiro lugar, o processo de inclusão de alunos com deficiência visual em aulas de EF na RR pode ser diferente entre as diversas regiões do país. Ao agrupar dados de alunos oriundos de múltiplos estados da federação, podemos ter incorrido em não identificar tendências mais ou menos inclusivas de certas localidades. Isto poderia ser solucionado com estudos de caso minuciosos que, ao comparar diferentes realidades, têm o potencial de identificar experiências de sucesso, barreiras e facilitadores para aumentar a participação desses alunos em aulas de EF. Em segundo lugar, cabe destacar que se trata de um estudo envolvendo somente jovens atletas de goalball, portanto, com certo grau de competência motora. Teoricamente, estes seriam física e, talvez, emocionalmente mais aptos a participar de atividades físicas durante as aulas de EF, o que pode ter superestimado nossos achados se considerarmos os alunos com deficiência visual que não praticam uma modalidade paralímpica. Nesse sentido, caberia investigar como se dá o processo de inclusão em situações reais dessa população durante as aulas de EF regular. Por fim, cabe destacar o caráter transversal deste estudo e a ausência de um grupo controle. Um estudo longitudinal com ambos os grupos permitiria examinar como se dá a inclusão de todo e qualquer aluno, independentemente de seu grau de habilidade e performance.

Concluímos que a participação de jovens com deficiência visual em aulas de EF na RR encontra-se inversamente relacionada ao grau de sua perda visual. Em decorrência da deficiência, foram elencados os seguintes fatores limitantes para a participação nas atividades 
desse contexto: a falta de colaboração dos colegas, o medo de se machucar, a falta de capacitação dos professores e as necessidades de adaptação do espaço. Embora não se alinhe ao paradigma da inclusão escolar, este estudo apontou ser a aula de EF ofertada em IE o local onde alunos com deficiência visual têm oportunidade de praticar esportes mais adequados a sua capacidade sensorial.

\section{Referências}

ALVES, M. L. T.; DUARTE, E. The participation of students with Down Syndrome in physical education classes: a case study. Movimento, v. 18, n. 3, p. 237-256, 2012.

Exclusion in physical education classes: factors associated with participation of students with disabilities. Movimento, v. 19, n. 1, p. 117-137, 2013.

A percepção dos alunos com deficiência sobre a sua inclusão nas aulas de Educação Física escolar: um estudo de caso. Revista Brasileira de Educação Física e Esportes, v. 28, n. 2, p. 329-338, 2014.

AMIRAliAn, M. L. T. et al. The concept of disability. Revista de Saude Pública, v. 34, n. 1, p. $97-103$, Feb. 2000.

BARTON, L. Disability, emporwement and physical education. In: EVANS, J. (Ed.). Equality, education and physical education. London: Falmer Press, 1993.

BETTI, M.; ZULIANI, L. R. Educação física escolar: uma proposta de diretrizes pedagógicas. Revista Mackenzie de Educação Física e Esporte, v. 1, n. 1, 2002.

BLINDE, E.; MCCALLISTER, S. Listening to the voices of students with physical disabilities: experiences in the Physical Education classroom. Journal of Physical Education, Recreation and Dance, v. 69, n. 6, p. 64-68, 1998.

BLOCK, M. E.; OBRUSNIKOVA, I. Inclusion in physical education: a review of the literature from 1995-2005. Adapted Physical Activity Quarterly, v. 24, n. 2, p. 103-124, Apr. 2007.

BOOTH, T.; AINSCOWN, M. Index for inclusion: developing learning and participation in schools. Centre for Studies in Inclusive Education. Bristol, UK: Centre for Studies in Inclusive Education, 2002.

BORGMANN, T.; ALMEIDA, J. J. G. Esporte paralímpico na escola: revisão bibliográfica. Movimento, v. 21, n. 1, 2015.

BRASIL. Lei de Diretrizes e Bases da Educação Nacional. LDB 9.394, de 20 de dezembro de 1996. 1996.

Decreto-lei no 3298. Brasília: MEC/SEESP. 1999.

Diretrizes Nacionais para a Educação Especial na Educação Básica. Brasília: MEC/SEESP, 2001a. 
Plano Nacional de Educação - PNE. Brasília: INEP, 2001b.

. Decreto no 186, de 9 julho de 2008. Aprova o texto da Convenção sobre os Direitos das Pessoas com Deficiência e de seu Protocolo Facultativo. 2008a.

Política Nacional de Educação Especial na Perspectiva da Educação Inclusiva. Brasília: MEC/SEESP, 2008b.

BREDAHL, A. M. Sitting and watching the others being active: the experienced difficulties in PE when having a disability. Adapted Physical Activity Quarterly, v. 30, n. 1, p. 40-58, Jan. 2013.

BRITTAIN, I. Perceptions of disability and their impact upon involvement in sport for people with disabilities at all levels. Journal of Sport \& Social Issues, v. 28, n. 4, p. 429-452, Nov. 2004a.

The role of schools in constructing self-perceptions of sport and physical education in relation to people with disabilities. Sport, Education and Society, v. 9, n. 1, p. 75-94, $2004 b$.

COATES, J.; VICKERMAN, P. Let the children have their say: children with special educational needs and their experiences of Physical Education - a review. Support for Learning, v. 23, n. 4, p. 168-175, 2008.

COSTA, C. de M.; MUNSTER, M. de A. V. Adaptações Curriculares nas Aulas de Educação Física Envolvendo Estudantes com Deficiência Visual. Revista Brasileira de Educação Especial, v. 23, n. 3, p. 361-376, 2017.

Curricular adaptations in physical education classes involving students with visual impairments. Revista Brasileira de Educação Especial, v. 23, n. 3, p. 361-376, 2017.

DEPAUW, K. The (in)visibility of disability: cultural contexts and "sporting bodies". Quest, v. 49, n. 4, p. 416-430, 1997.

EVANS, J. Making a difference? education and 'ability' in physical education. European Physical Education Review, v. 10, n. 1, p. 95-108, 2004.

FIORINI, M. L. S.; NABEIRO, M. Um estudo sobre a intervenção com o professor de Educação Física para inclusão educacional do aluno com deficiência visual. Revista Brasileira de Atividade Motora Adaptada, v. 14, n. 2, p. 21-26, 2013.

FITZGERALD, H. Still feeling like a spare piece of luggage? embodied experiences of (dis)ability in physical education and school sport. Physical Education and Sport Pedagogy, v. 10, n. 1, p. 41-59, 2005.

FURTADO, O. L. P. C. et al. Physical activity interventions for children and youth with visual impairments. Adapted Physical Activity Quarterly, v. 32, n. 2, p. 156-176, 2015. 
et al. Health-related physical fitness among young goalball players with visual impairments. Journal of Visual Impairment \& Blindness, v. 110, n. 4, p. 257, 2016.

GOODWIN, D. L.; WATKINSON, E. J. Inclusive physical education from the perspective of students with physical disabilities. Adapted Physical Activity Quarterly, v. 17, n. 2, p. 144160, Apr. 2000.

GRENIER, M.; COLLINS, K.; WRIGHT, S. Perceptions of disability sports unit in general physical education. Adapted Physical Activity Quarterly, v. 31, p. 49-66, 2014.

HAEGELE, J. A.; SUTHERLAND, S. Perspectives of students with disabilities toward physical education: a qualitative inquiry review. Quest, v. 67, n. 3, p. 255-273, Jul. 2015.

HAYNES, S. N.; RICHARD, D.; KUBANY, E. S. Content validity in psychological assessment: a functional approach to concepts and methods. Psychological Assessment, v. 7, n. 3, p. 238-247, 1995.

INEP. Censo Escolar do Instituto Nacional de Estudos e Pesquisas Educacionais. Brasília, 2012. Disponível em:

<http://portal.inep.gov.br/web/guest/sinopses-estatisticas-da-educacao-basica>. Acesso em: 23 fev. 2018.

KARAKAYA, I. C.; AKI, E., \& ERGUN, N. Physical fitness of visually impaired adolescent goalball players. Perceptual and Motor Skills, v. 108, n. 1, p. 129-136, 2009.

KODISK, S.; KULINNA, P. H.; MARTIN, J.; PANGRAZ, K.; DARST, P. Determinants of physical activity in an inclusive setting. Adapted Physical Activity Quarterly, v. 23, p. 390409, 2006.

MARCHESI, A. A. Prática das escolas inclusivas. In: RODRIGUES, D. (Ed.). Educação e diferença: valores e práticas para uma educação inclusiva. Portugal: Porto, 2001. p. 93-108.

MILES, M. B.; HUBERMAN, A. M.; SALDAÑA, J. Qualitative data analysis: a methods sourcebook. Thousand Oaks, CA: SAGE, 2014.

MITTLER, P. Educação inclusiva: contextos sociais. Porto Alegre: Artmed, 2003.

MUNSTER, M. A. V.; ALMEIDA, J. J. G. Atividade física e deficiência visual. In: GORGATI, M. G.; C., R. F. Atividade física adaptada: qualidade de vida para pessoas com necessidade especiais. Barueri, São Paulo: Monole, 2005. p. 33-51.

ODOM, S. L.; DIAMOND, K. E. Inclusion of young children with special needs in early childhood education: the research base. Early Childhood Research Quarterly, v. 13, n. 1, p. $3-25,1998$.

PIVIK, J.; MCCOMAS, J.; LAFLAME, M. Barriers and facilitators to inclusive education. Exceptional Children, Virginia, v. 69, n. 1, p. 97-107, 2002. 
PLACE, K.; HODGE, S. R. Social inclusion of students with psysical disabilities in general physical education: a behavioral analyses. Adapted Physical Activity Quartely, v. 18, 389404, 2001.

QI, J.; HA, A. S. Inclusion in Physical Education: a review of literature. International Journal of Disability Development and Education, v. 59, n. 3, p. 257-281, 2012.

RODRIGUES, D. A. Educação e a diferença. In: RODRIGUES, D. A. (Ed.). Educação e diferença: valores e práticas para uma educação inclusiva. Portugal: Porto Editora, 2001.

SAGE, G. H. Sport and physical education and the new world order: dare we be agents of social change? Quest, v. 45, p. 151-164, 1993.

SPENCER-CAVALIERE, N.; WATKINSON, E. J. Inclusion understood from the perspectives of children with disability. Adapted Physical Activity Quarterly, v. 27, n. 4, p. 275-293, Oct. 2010.

STAINBACK, W.; STAINBACK, S. Colaboração, rede de apoio e construção de comunidade. IN: STAINBACK, W.; STAINBACK, S. Inclusão: um guia para educadores. Porto Alegre: Artes Médicas, 1999.

STEWART, N. W. et al. Considerations for conducting imagery interventions in physical education settings. Journal of Imagery Research in Sport and Physical Activity, v. 10, n. 1, p. 31-47, 2015.

WHO. Global recommendations on physical activity for health. Geneva, 2010. Disponível em: <http://www.who.int/dietphysicalactivity/publications/9789241599979/en/>. Acesso em: 23 fev. 2018.

Recebido em: 26/02/2018

Revisado em: 27/06/2018

Aprovado em: 19/11/2018

Endereço para correspondência:

otaviofurtado@hotmail.com

Otávio Luis Piva da Cunha Furtado

Universidade de São Paulo, Escola de Educação Física e Esporte de Ribeirão Preto

Av. Bandeirantes $n^{\circ} 3900$

Monte Alegre

14040-900 - Ribeirão Preto - SP 